\title{
Expérience française de balance du watt : conception d'un fléau de balance monobloc et étude de son comportement mécanique
}

\author{
Patrick Pinot ${ }^{1, a}$, Yang Liu ${ }^{1}$, Patrick Espel ${ }^{2}$, Matthieu Thomas ${ }^{2}$, Djamel Ziane ${ }^{2}$, François Piquemal ${ }^{2}$ \\ ${ }^{1}$ Conservatoire national des arts et métiers, Laboratoire commun de métrologie, 61 rue du Landy 93210 La Plaine Saint-Denis, France \\ ${ }^{2}$ Laboratoire national de métrologie et d'essais, Laboratoire commun de métrologie, 29 avenue Roger Hennequin 78197 Trappes cedex \\ France
}

\begin{abstract}
Since 2005, the French watt balance experiment, installed at the LNE, has been using a balance beam with removable flexure hinges designed at the CNAM. With this balance, a first determination of the Planck constant in air was performed in 2014. This balance beam presents some drawbacks. First, it is difficult to mount and adjust the hinges. Secondly, the use of clamped flexure strips used as pivots limits the beam sensitivity and measurement repeatability, moreover the central flexure strip twists under the effect of unwanted horizontal forces at the ends of the beam, which leads to a potential measurement error. For these reasons, a new, single-piece balance beam has been designed. It is not only easier to mount and adjust than its predecessor, but also free of unwanted stresses such as shear force on the hinges. This ensures that, once the beam is loaded, the rotation axes are by construction parallel and coplanar. In addition, the use of a double central hinge strongly reduces the torsion effect.
\end{abstract}

\section{Introduction}

L'expérience de la balance du watt repose sur un principe de puissance virtuelle pour comparer indirectement une puissance mécanique et une puissance électrique. L'idée a été proposée en 1975 par l'anglais B.P. Kibble [1]. L'expérience se déroule en deux phases :

- l'une, dite statique, dans laquelle un conducteur de longueur $l$ parcouru par un courant $I$ est placé dans un champ d'induction homogène $B$ de telle sorte que la force de Laplace $F_{e}$ s'exerçant sur celui-ci soit verticale. Le courant $I$ est ajusté pour que la force sur le conducteur, suspendu à un fléau de balance, équilibre le poids $P$ d'une masse $m$ soumise à l'accélération de la pesanteur $g$. Moyennant des hypothèses d'orthogonalité et de configuration géométrique parfaite, l'équilibre de la balance se traduit par l'équation :

$$
P=F_{e} \quad \text { ou encore } \quad m g=B I I
$$

- la seconde phase est dite dynamique. Pour déterminer expérimentalement le produit $B l$ avec l'exactitude recherchée, elle consiste à déplacer à une vitesse $v$, le même conducteur en boucle ouverte dans le même champ magnétique $B$ suivant une trajectoire verticale. L'expérience requiert seulement l'invariance de l'induction $B$, de la longueur $l$ du conducteur pendant le temps de la mesure et de la position relative de la bobine par rapport au champ d'induction. En posant là encore des hypothèses d'orthogonalité et de configuration géométrique parfaite, la force électromotrice induite $U$ aux bornes du conducteur s'écrit :

$$
U=v B l
$$

De cette expression, on déduit que le produit $B l$ équivaut à $U / v$. Par substitution dans l'équation (1), on arrive à une équation d'égalité entre puissance mécanique et puissance électrique :

$$
m g v=U I
$$

Le courant $I$ est mesuré par la différence de potentiel $V$ qu'il provoque aux bornes d'une résistance $R$. L'expression (3) peut alors s'écrire :

$$
m g v=U V / R
$$

Les tensions $V$ et $U$ sont déterminées par comparaison à une tension de référence $U_{J}$ délivrée par un étalon à effet Josephson et la résistance $R$ par comparaison à une résistance $R_{H}$ d'un étalon quantique de résistance basé sur l'effet Hall quantique. Ces deux effets quantiques macroscopiques permettent de rattacher les valeurs de tension et de résistance électriques à deux constantes : la constante de Josephson $K_{\mathrm{J}}$ et la constante de von Klitzing $R_{\mathrm{K}}$ qui sont reliées à la constante de Planck $h$ et à la charge élémentaire $e$ par les deux relations théoriques $K_{\mathrm{J}}$ $=2 e / h$ et $R_{\mathrm{K}}=h / e^{2}$. Ces relations appliquées à l'expression de la puissance électrique permettent d'éliminer $e$, ne laissant apparaître que la constante de Planck $h$ facteur d'un paramètre expérimental $A$ [2] :

$$
m g v=A h / 4
$$

\footnotetext{
a patrick.pinot@cnam.fr
} 
Depuis 2002, la France développe une expérience de balance du watt, installée au Laboratoire national de métrologie et d'essais (LNE Trappes). L'induction magnétique $B$ dans l'entrefer de l'aimant permanent de l'expérience est de l'ordre de $1 \mathrm{~T}$ et la longueur $l \mathrm{du}$ fil du circuit magnétique est d'environ $600 \mathrm{~m}$. La source de courant permet d'engendrer une force de Laplace de2,5 N maximum. L'étalon de masse $m$ choisi pour l'expérience a une masse nominale de $500 \mathrm{~g}$. Une première détermination de la constante de Planck $h$ dans l'air au moyen de l'expérience française a été réalisée en 2014 [3]. La valeur de $h$ extraite des données obtenues avec cette expérience et son incertitude-type associée sont :

$$
h=6,6260688(20) \times 10^{-34} \mathrm{~J} . \mathrm{s}
$$

Ce qui correspond à une incertitude type relative de $3,1 \times 10^{-7}$.

Cette valeur diffère en terme relatif de la valeur recommandée CODATA 2010 de $1,1 \times 10^{-7}$. L'objectif pour la métrologie française est de déterminer la constante de Planck avec une incertitude type relative de $5 \times 10^{-8}$.

En 2005, le Conservatoire national des arts et métiers (CNAM) a conçu, développé, usiné et caractérisé le fléau à pivots flexibles utilisé actuellement sur l'expérience. Le comportement des articulations flexibles est bien connu [4-8] et leur utilisation comme pivot pour les balances a été étudiée $[9,10]$. Ce genre d'articulations est couramment mis en œuvre dans les meilleurs comparateurs de masse.

En 2015, à partir de 1'expérience acquise sur la technologie des fléaux de balance et suite aux nombreuses caractérisations statiques et dynamiques réalisées au CNAM et au LNE (stabilité, sensibilité, influence de la position du centre de gravité, étude des mouvements de tangage, roulis et lacet du fléau ...), le CNAM a entrepris la conception d'un nouveau fléau monobloc. Il a étudié son comportement mécanique virtuellement par simulation et expérimentalement sur un montage similaire à un électrodynamomètre. Dans cet article, on montre que ce nouveau prototype de fléau devrait offrir une meilleure stabilité (plus faible sensibilité aux forces parasites et meilleure répétabilité des mesures) que le prototype actuel. Il sera mis en œuvre sur l'expérience française pour les prochaines mesures de la constante de Planck réalisées dans l'air ou sous vide.

\section{Conception d'un fléau monobloc}

\subsection{Motivation}

Le CNAM a mis au point en 2005 un prototype de fléau à pivots flexible. Ce prototype est constitué d'une poutre en alliage d'aluminium au centre et aux extrémités de laquelle des éléments de fixation amovibles pour lames flexibles sont positionnés au moyen d'un système de Boyle (système de 3 liaisons mécaniques de type bille/Vé, les trois Vés étant équidistants dans un même plan et orientés à $120^{\circ}$ les uns par rapport aux autres).Les lames flexibles, feuilles d'acier inoxydable de $20 \mu \mathrm{m}$ d'épaisseur et $20 \mathrm{~mm}$ de large sont maintenues à chacune de leurs extrémités par pincement entre mors.

Bien que ce prototype permette d'obtenir une sensibilité du fléau satisfaisante et des mesures en phase statique avec une répétabilité relative de $1^{\prime}$ ordre de $10^{-8}[11]$, il présente plusieurs inconvénients tels que :

- la difficulté pour définir et régler la position des centres de rotation (parallélisme, coplanarité sous charge) ;

- 1'apparition de contraintes au bord des mors (discontinuité de forme et de matière, force de cisaillement, glissement) ;

- l'apparition d'un phénomène de torsion de la lame centrale sous l'effet de forces parasites horizontales transverses agissant aux extrémités du fléau ;

- la difficulté de montage entre mors des lames et d'installation du fléau sur la structure de la balance.

En 2014, pour résoudre ou, à tout le moins, atténuer ces inconvénients et répondre aux exigences techniques formulées par les collègues du LNE travaillant sur l'expérience de balance du watt, le CNAM a conçu un nouveau prototype de fléau de type monobloc.

\subsection{Conception}

Afin de garantir le parallélisme et la coplanarité des axes de rotation sous charge, il a été décidé de réaliser un fléau monobloc, c'est-à-dire ayant son pivot central et ses deux pivots d'extrémité usinés dans le bloc constituant la poutre maîtresse du fléau.

Pour définir aussi précisément que possible la position des axes de rotation, des articulations à profil circulaire symétrique ont été choisies (voir figure 1).

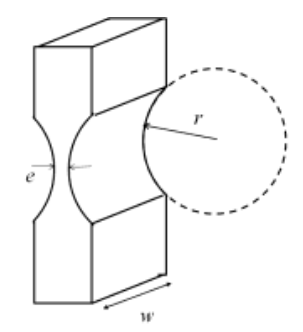

Figure 1. Articulation flexible à profil circulaire symétrique.

La sensibilité d'un fléau étant inversement proportionnelle à sa masse et proportionnelle à la longueur de ses bras, il y a donc un compromis à trouver entre faible masse et long bras. Cela a conduit à choisir une longueur de bras de $100 \mathrm{~mm}$ pour une masse du fléau d'environ $150 \mathrm{~g}$ en choisissant un alliage d'aluminium pour sa réalisation.

De plus, ses articulations doivent être alignées sous charge et avoir une faible rigidité en flexion. Il faut donc un alliage d'aluminium ayant de bonnes caractéristiques mécaniques particulièrement en termes d'élasticité.

Pour toutes ces raisons, l'alliage EN AW-7075 T651 a été retenu pour cette réalisation. 
L'usinage des pivots flexibles à profil circulaire symétrique ne peut se faire que par électro-érosion. Il est connu que cette technique d'usinage altère superficiellement la structure du matériau sur une profondeur de l'ordre de $10 \mu \mathrm{m}$ [12 - 14]. Cela signifie qu'à l'épaisseur du col déterminée théoriquement pour une largeur et une charge données, il sera nécessaire d'ajouter $20 \mu \mathrm{m}$. Dans la configuration actuelle de la balance du watt, la charge supportée par chaque pivot d'extrémité est d'environ $30 \mathrm{~N}$ et celle supportée par le double pivot central est un peu plus du double puisqu'il y a, en plus, la charge correspondant à la masse du fléau lui-même et des éléments fixés sur le fléau. On obtient ainsi une épaisseur au col de l'articulation de $40 \mu \mathrm{m}$ pour une largeur de $10 \mathrm{~mm}$ sous une charge de $30 \mathrm{~N}$.

Enfin, pour limiter les effets de torsion du pivot central, cette articulation a été doublée de manière à augmenter sa rigidité en torsion.

La figure 2 présente le schéma de position des articulations dans un plan horizontal et donne les principales dimensions caractéristiques de leur disposition.

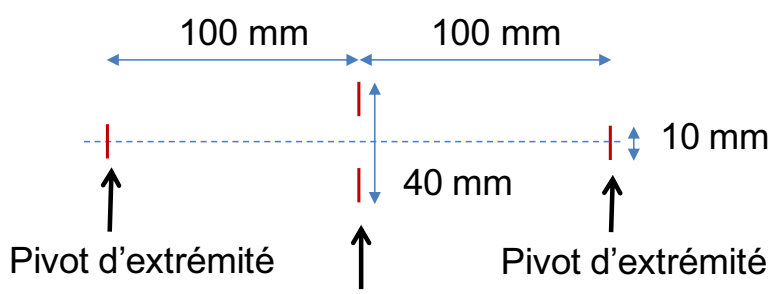

\section{Double pivot central}

Figure 2. Position des pivots du fléau monobloc (vue de dessus)

Il faut également concevoir la plaque de fixation du pivot central à la structure de la balance du watt ainsi que la liaison des pivots d'extrémités aux suspensions des différentes charges (bobine et plateaux pour la masse et la tare). Ces éléments doivent être usinés dans le même bloc de matière en continu avec le fléau. Enfin, les pièces de bridage des pivots doivent être conçues de manière à facilité l'installation et la mise en œuvre du fléau.

Il faut aussi prévoir les emplacements pour la fixation sur le fléau de pièces telles que vis pour masselotte de réglage du centre de gravité du fléau, support pour le passage de fils électriques, support pour les composants optiques de détection...

La figure 3 donne une représentation en perspective du fléau avec les principaux éléments mécaniques qui doivent y être fixés.

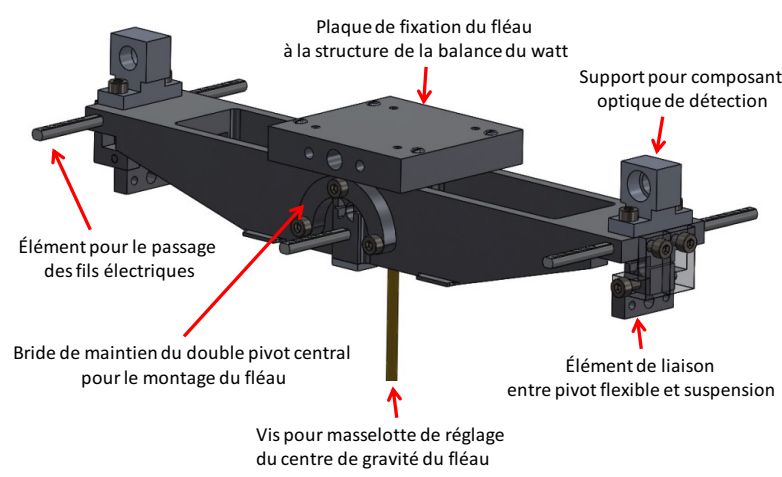

Figure 3. Représentation en perspective du fléau monobloc.

\subsection{Simulation}

Préalablement à la mise en fabrication du fléau, il a été nécessaire de faire des calculs et des simulations de comportement mécanique de sa structure pour déterminer, entre autres, les zones de contraintes, les valeurs limites de rupture et d'élasticité, la flexion des bras sous charge.

En premier lieu, il faut estimer la charge suspendue à chaque extrémité du fléau pour évaluer les contraintes et déplacements.

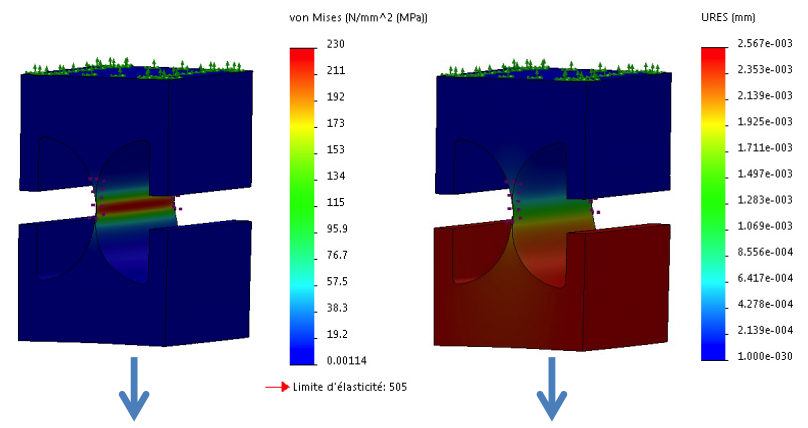

Figure 4. Exemple de simulation sur l'articulation à profil circulaire symétrique réalisée en EN AW-7075 T651 de $20 \mu \mathrm{m}$ au col et $10 \mathrm{~mm}$ de large sous une charge de $50 \mathrm{~N}$ :

- à gauche en termes de contrainte de von Mises ;

- à droite en termes de déplacement.

La figure 4 donne un exemple de simulation sur une articulation à profil circulaire symétrique de $20 \mu \mathrm{m}$ au col (correspondant à l'épaisseur non altérée par l'électroérosion) et $10 \mathrm{~mm}$ de large sous une charge de $50 \mathrm{~N}$. A gauche, il s'agit d'une simulation en termes de contrainte de von Mises, on obtient une valeur maximale au col de $230 \mathrm{MPa}$ et, à droite, en termes de déplacement dont le maximum est de $2,5 \mu \mathrm{m}$. Ces résultats montrent, d'une part, que la contrainte au col de l'articulation reste inférieure à sa limite d'élasticité (505 $\mathrm{MPa})$ même pour une charge supérieure de $20 \mathrm{~N}$ à la charge réelle et, d'autre part, que pour cette charge de simulation de $50 \mathrm{~N}$, l'articulation s'allonge autour du col d'une valeur suffisamment faible pour pouvoir être compensée par les réglages mécaniques du fléau. 


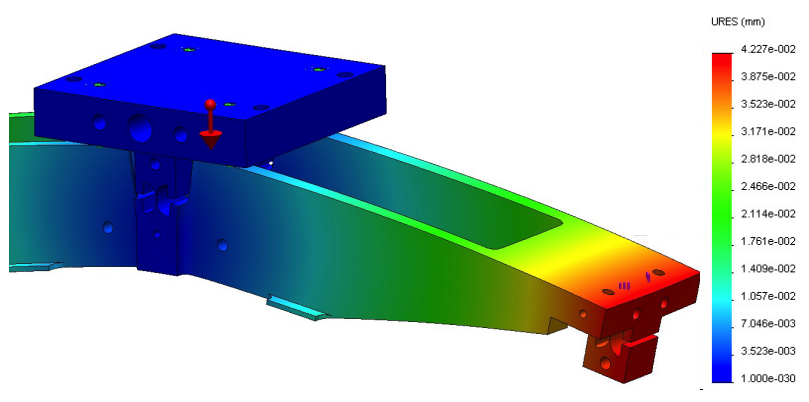

Figure 5. Exemple de simulation de déplacement en flexion du bras de fléau sous $30 \mathrm{~N}$ de charge appliquée au niveau du pivot d'extrémité.

La figure 5 présente un exemple de simulation de flexion de bras sous charge de $30 \mathrm{~N}$ appliquée au niveau des pivots d'extrémité. Dans cet exemple, on observe une flexion de l'ordre de $40 \mu \mathrm{m}$ au col, ce qui conduirait à faire usiner le col du double pivot central $40 \mu \mathrm{m}$ en dessous du plan défini par les axes de rotation des deux pivots d'extrémité pour obtenir l'alignement des axes de rotation sous une charge d'équilibre de $30 \mathrm{~N}$.

Ce travail de simulation a permis d'affiner les plans du fléau et des différentes pièces adaptées au fléau.

\subsection{Réalisation}

Divers essais d'usinage des articulations à profil circulaire symétrique par électro-érosion au fil ont été effectués. En particulier pour le double pivot central dont la largeur totale (profondeur) est de $40 \mathrm{~mm}$, il était nécessaire de vérifier qu'il n'y ait pas de phénomène d'élancement qui se traduit par une variation de l'épaisseur du col sur sa profondeur. Ce phénomène a été observé sur des échantillons usinés par l'atelier du LNE. Cela provient d'un défaut de parallélisme du fil d'électroérosion entre l'usinage d'un côté et la reprise de l'autre côté de l'articulation, provoquant un amincissement de l'épaisseur du col tout le long de sa profondeur, le fil peut finir par traverser l'épaisseur du col à l'une des extrémités de l'articulation.

Suite à des premiers résultats non satisfaisants, nous avons recherché une entreprise capable de maîtriser ce phénomène.

Deux prototypes de fléau monobloc ont alors été usinés en alliage d'aluminium EN AW-7075 T651 par l'entreprise VUILLERMOZ Philippe SAS. L'un étant réservé aux essais de validation de la conception et l'autre devant être implanté sur la balance du watt après qualification métrologique.

\subsection{Qualification}

L'examen des prototypes usinés a révélé pour l'un d'eux un défaut à l'une des extrémités du double pivot central au niveau du col.

Bien que l'on soit proche d'un phénomène d'élancement, il s'agit plutôt d'un arrachement de matière, peut-être dû à un défaut local de structure du matériau. Ce défaut n'étant pas rédhibitoire pour utiliser ce prototype, nous avons choisi de l'installer sur un banc d'essai sur lequel il sera soumis à des tests statiques et dynamiques sous charge. Ce banc est semblable au montage de la phase statique de la balance du watt, mais il ne peut équilibrer qu'une force plus faible puisqu'il comporte un aimant permanent dont le champ d'induction n'est que de 0,2 T. La figure 6 présente une photographie de ce prototype monté sur le banc d'essais. Ces essais permettront également de caractériser ses qualités métrologiques en termes de sensibilité, mobilité, stabilité et répétabilité.

Les premiers tests expérimentaux qui sont en cours n'ont pas révélé d'anomalie comportementale du prototype.

Dans le cas probable où les résultats des essais de validation et de qualification seraient satisfaisants, le second prototype sera installé sur l'expérience française de balance du watt pour effectuer de nouvelles déterminations de la constante de Planck réalisées dans l'air ou sous vide.

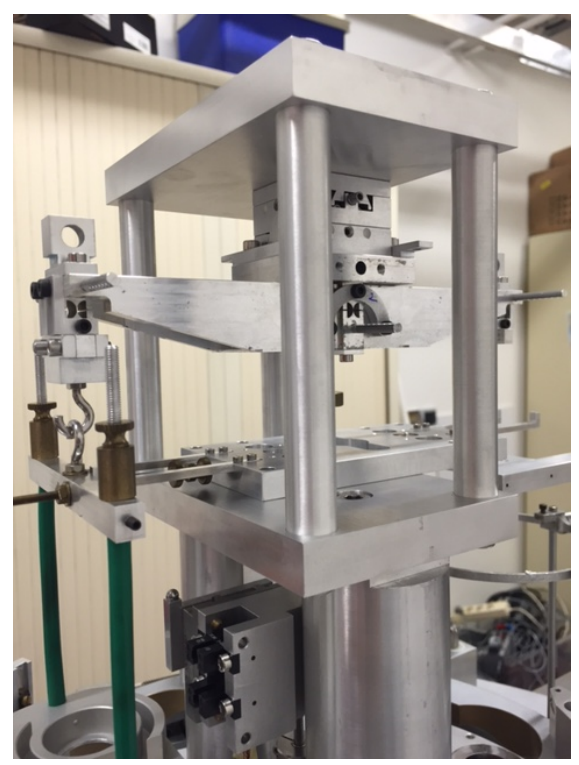

Figure 6. Photographie de la partie supérieure du banc d'essais montant l'un des prototypes de fléau monobloc en cours de montage sur ce banc.

\section{Conclusion}

Le nouveau prototype de fléau monobloc conçu et développé par le CNAM pour être monté sur l'expérience de balance du watt installé au LNE (Trappes) permet d'éliminer certains inconvénients que présente le fléau qui a été utilisé jusqu'à maintenant.

Les premiers résultats de tests sont encourageants. Ce nouveau fléau devrait permettre d'obtenir une meilleure stabilité et répétabilité des mesures pour la phase statique de l'expérience.

Cependant la sensibilité mécanique du fléau devrait être un peu moins bonne que celle obtenue avec le fléau précédent en raison de la plus grande rigidité des pivots flexibles. Cet inconvénient devrait pouvoir être compensé par la résolution suffisamment élevée du système de détection de la position du fléau [15].

L'inconvénient majeur de cette conception de fléau monobloc est qu'en cas de rupture de l'une des articulations, il faut remplacer entièrement le fléau. Ce risque est atténué par le fait que le coefficient de sécurité 
par rapport à la charge limite de rupture est de l'ordre de 6 au lieu de 3 sur l'ancien fléau.

"This work was funded through the European Metrology Research Programme (EMRP) Project SIBO3 $\mathrm{kNOW}$. The EMRP is jointly funded by the EMRP participating countries within EURAMET and the European Union."

\section{Références}

1. B P Kibble, Atomic Masses and Fundamental Constants (5th edn ed J H Sanders and A H Wapstra, New York: Plenum; 1976) pp 545-51

2. A. Eichenberger, G. Genevès, P. Gournay, Eur. Phys. J. Spec. Top. 172 363-83 (2009)

3. M Thomas, P Espel, D Ziane, P Pinot, P Juncar, F Pereira Dos Santos, S Merlet, F Piquemal, G Genevès, Metrologia 52 433-443 (2015).

4. F.S. Eastman, J. Aeronautical Science, 5, 1937, 16-21.

5. W. H. Wittrick, Aust. J. Sci. Res. A, 1, 1948, 121-134.

6. W. H. Wittrick, Aeronautical Quaterly, 11, 1951, 272-292.

7. W.D.Weinstein, Machine Design, 37, 1965, 136-145.

8. P. H. Sydenham, J. Phys. E: Sci. Instrum., 17, 1984, 922-930.

9. A. Picard, Metrologia, 41, 2004, 319-329.

10. C. C. Speake, Proc. R. Soc. London, Ser. A 414, 333 (1987).

11. M Thomas, P Espel, D Ziane, P Pinot, P Juncar, F Pereira Dos Santos, S Merlet, F Piquemal, G Genevès, Metrologia 52 433-443 (2015).

12. P. Pinot, G. Genevès, EPJ Web of Conferences 77, 00005 (2014).

13. S. Henein, «Conception des guidages flexibles», Presses Polytechniques et Universitaires Romandes (2004)

14. P. Merken and J. F. Debongnie, Proceeding of the $6^{\text {th }}$ National Congress on Theoretical and Applied Mechanics, Ghent, NCTAM-2003-077 (2003).

15. Haddad D., Juncar P., Geneves G. and Wakim M., IEEE, Trans. Instrum. Meas. 58 1003-9 (2009). 